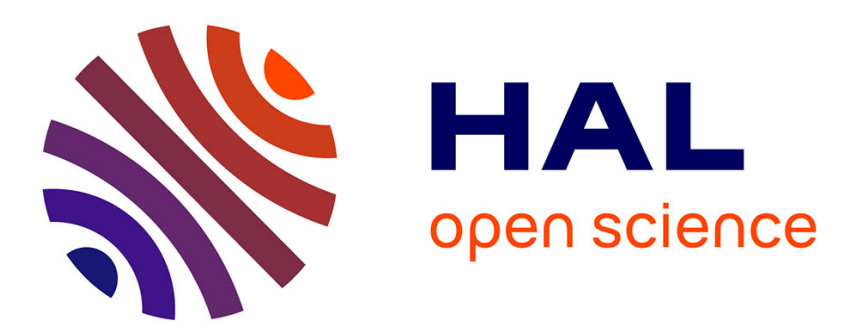

\title{
Experimental comparison of velocity estimators for a control moment gyroscope inverted pendulum
}

Dmitry Sokolov, Stanislav Aranovskiy, Alexander A Gusev, Igor Ryadchikov

\section{To cite this version:}

Dmitry Sokolov, Stanislav Aranovskiy, Alexander A Gusev, Igor Ryadchikov. Experimental comparison of velocity estimators for a control moment gyroscope inverted pendulum. AMC 2020 - 16th IEEE International Workshop on Advanced Motion Control, Sep 2020, Kristiansand / Virtual, Norway. hal-02313600v2

\section{HAL Id: hal-02313600 https://hal.inria.fr/hal-02313600v2}

Submitted on 7 Nov 2019

HAL is a multi-disciplinary open access archive for the deposit and dissemination of scientific research documents, whether they are published or not. The documents may come from teaching and research institutions in France or abroad, or from public or private research centers.
L'archive ouverte pluridisciplinaire HAL, est destinée au dépôt et à la diffusion de documents scientifiques de niveau recherche, publiés ou non, émanant des établissements d'enseignement et de recherche français ou étrangers, des laboratoires publics ou privés. 


\section{Experimental comparison of velocity estimators for a control moment gyroscope inverted pendulum}

\author{
Dmitry Sokolov \\ Université de Lorraine, CNRS, Inria, LORIA \\ F-54000 Nancy, France \\ dmitry.sokolov@univ-lorraine.fr
}

\author{
Alexander Gusev \\ Kuban State University \\ Krasnodar, Russia \\ gusev@ftf.kubsu.ru
}

\author{
Stanislav Aranovskiy \\ Equipe Automatique, CentaleSupélec - IETR \\ Cesson-Sévigné, France \\ stanislav.aranovskiy@centralesupelec.fr \\ Igor Ryadchikov \\ Kuban State University \\ Krasnodar, Russia \\ ryadchikov@kubsu.ru
}

\begin{abstract}
We consider the problem of velocity estimation for a control moment gyroscope inverted pendulum. To this end, we a consider model-free differentiator, a model-based linear observer and a model-based nonlinear differentiator. The proposed designs are implemented in hardware and the closedloop system performance in the stabilization task is compared. Moreover, we show that the considered system cannot be partially linearized via a change of coordinates, and thus is not suitable for a recently reported class of nonlinear observers.

Index Terms-velocity estimation, differentiator, inverted pendulum, control moment gyroscope
\end{abstract}

\section{Introduction}

The research problem of this paper is motivated by the walking robot we are currently developing in the Laboratory of Robotics and Mechatronics of the Kuban State University (refer to Figure 11. left). This nonanthropomorphic robot has an auxiliary dynamic stabilization system which consists of two scissored pairs of control moment gyroscopes (CMG). The scissored pairs are orthogonal and thus the problem of vertical stabilization of the robot can be considered for each axis separately. Therefore, stabilization of the robot for one axis can be approximated with a simplified onedimensional prototype. In this paper we consider such a prototype, which is a control moment gyroscope inverted pendulum (Figure 22). Note that the robot has a modular design: the biped is equipped with four identical CMG cubes.

Control moment gyroscope is a widely used technological device that uses the reaction of a spinning wheel to external torques. Due to the advantages of a large ratio of produced torque to control torque and relatively low power consumption, CMGs have a wide range of applications, including vessel stabilisation [1], motorcycle and robot balancing [2], balancing aid for humans and

This work was supported by the Russian Ministry of Education and Science, through the grant No. 8.2321.2017. bipedal exoskeletons [3], [4], attitude steering system for the satellites [5] and underwater vehicles [6].

The key element for stabilization of an inverted pendulum is velocity estimation. Note that our robot uses multiple accelerometers to estimate its tilt angles 7 , and we rely on soft sensors for velocity estimation.

Velocity estimation for a mechanical system is a wellknown problem, see, for example, the recent work by Aranovskiy et al. 8 and the references therein. In practice, a common solution is to consider this problem for each degree of freedom separately rather than to estimate all velocities with a single observer. From the signal processing point of view, this approach can be considered as numerical differentiation, where velocity estimation is seen as online differentiation of a measured position signal, e.g., a first-order difference used in [9]. While differentiator-based velocity observers can be designed model-free, a better performance is typically obtained when observers use (at least partially) available model knowledge: sliding-mode exact differentiators first proposed by Levant in [10], highgain differentiators as described by Vasiljevic and Khalil in [11, and differentiators proposed by Perruquetti et al. 112] that ensure finite-time convergence and robustness with respect to measurement noises and uncertainties. In this paper, we aim at practical comparison of some of the discussed observers.

The contribution of this paper is two-fold: first, we show that, despite its simplicity, the mechanical system under consideration does not belong to the class of recently reported systems [8], for which a construction of modelbased nonlinear observers is known. Next, we propose a comparison of three velocity observers, namely a modelfree differentiator, a model-based linear observer and a model-based nonlinear differentiator. All three observers are tested on the hardware we have built for this purpose, and the experimental results are provided.

The rest of the paper is organized as follows. In section II we present a model of the considered system. Next, in 


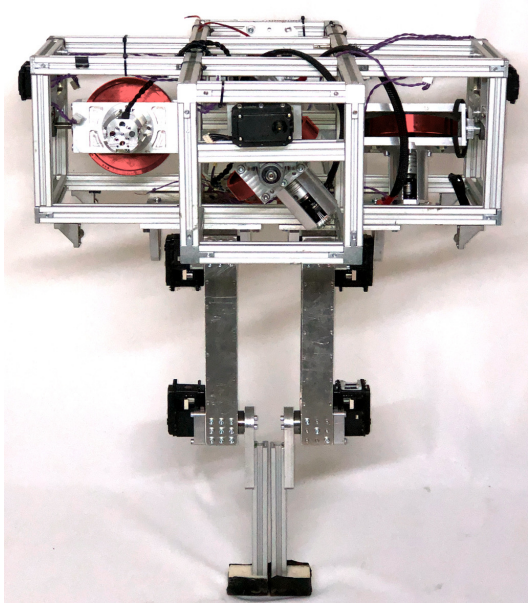

Fig. 1: We are developing a biped that uses four control moment gyroscopes (highlited in red) as an auxiliary stabilization system.
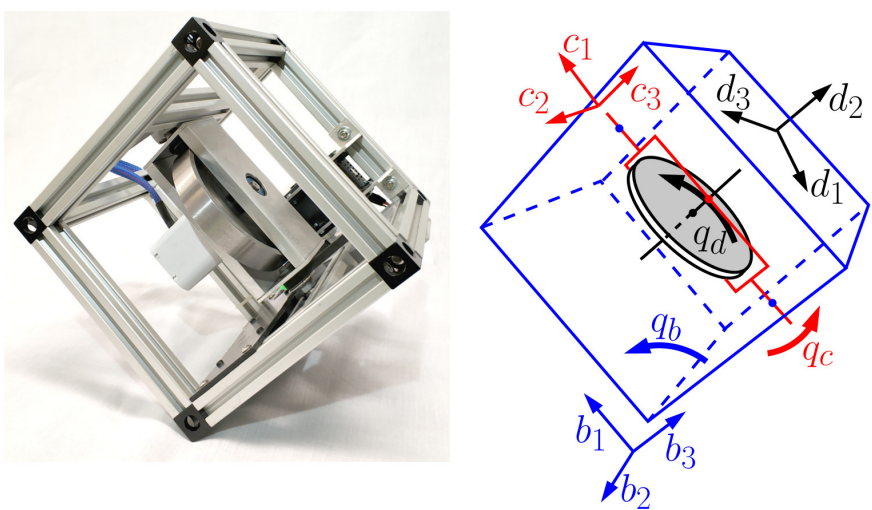

Fig. 2: 1D pendulum hardware and corresponding notations. This configuration corresponds to $q_{b}=-\pi / 4$ and $q_{c}=\pi / 2$.

section III we present the state-feedback controller capable to stabilize the system if all states (including velocities) are measured. Then, in section IV we discuss three velocity observers. Hardware experiments and observers comparison are provided in section $\mathrm{V}$ Finally, possible further research directions are discussed in the concluding section VI.

\section{Model Description}

The considered inverted pendulum is shown in Figure 2 . In this paper we follow the notations from the manual of the model 750 control moment gyroscope commercialized by Educational Control Products company [13. The notations and the corresponding hardware parameters are summarized in the Table [1. The pendulum consists of three bodies: the main body $B$, the gimbal $C$, and the wheel $D$. We associate a frame with each of the bodies: $\left\{O, \vec{b}_{1}, \vec{b}_{2}, \vec{b}_{3}\right\},\left\{O, \vec{c}_{1}, \vec{c}_{2}, \vec{c}_{3}\right\}$, and $\left\{O, \vec{d}_{1}, \vec{d}_{2}, \vec{d}_{3}\right\}$, respectively. We assume that all the bodies are symmetric, and the centers of mass of all the bodies coincide at the point $O$. Due to the symmetry, the inertia matrices are diagonal. We denote the principal moments of inertia (w.r.t. the center of mass) of the bodies $B, C$ and $D$ as $\operatorname{diag}\left(I_{b}, J_{b}, K_{b}\right), \operatorname{diag}\left(I_{c}, J_{c}, K_{c}\right)$ and $\operatorname{diag}\left(I_{d}, J_{d}, K_{d}\right)$, respectively.

The angle of the body $B$ with respect to the vertical is denoted as $q_{b}$, the angle of the body $C$ with respect to the body $B$ is denoted as $q_{c}$, and the angle of the body $D$ with respect to the body $C$ is denoted as $q_{d}$. The configuration shown in Figure 2 corresponds to the angles $q_{b}=-\pi / 4$ and $q_{c}=\pi / 2$. Note that the equilibrium position of an actual robot depends on the configuration of the legs and may be subject to external disturbances; therefore, for our cube we model the equilibrium point as $q_{b}=-\pi / 4-e$, where $e$ is the unknown (small) bias.

The corresponding time derivatives are denoted as $\omega_{b}(t):=\dot{q}_{b}(t), \quad \omega_{c}(t):=\dot{q}_{c}(t), \quad \omega_{d}(t):=\dot{q}_{d}(t)$. If we apply torques $\tau_{c}$ and $\tau_{d}$ to the bodies $C$ and $D$, respectively, we can find the Euler-Lagrange equations:

$$
\begin{aligned}
0= & \left(J_{1}+J_{2} \cos ^{2} q_{c}\right) \dot{\omega}_{b}-J_{2} \sin \left(2 q_{c}\right) \omega_{c} \omega_{b} \\
& +J_{d} \dot{\omega}_{d} \cos q_{c}-J_{d} \sin \left(q_{c}\right) \omega_{c} \omega_{d} \\
& -m l g \sin \left(q_{b}+\frac{\pi}{4}+e\right) \\
\tau_{c}= & \left(I_{c}+I_{d}\right) \dot{\omega}_{c}+J_{d} \omega_{b} \omega_{d} \sin q_{c}+\frac{1}{2} J_{2} \sin \left(2 q_{c}\right) \omega_{b}^{2}, \\
\tau_{d}= & J_{d} \dot{\omega}_{d}+J_{d} \cos \left(q_{c}\right) \dot{\omega}_{b}-J_{d} \sin \left(q_{c}\right) \omega_{c} \omega_{b},
\end{aligned}
$$

where $J_{1}=I_{d}+J_{b}+K_{c}+m l^{2}, J_{2}=J_{c}-I_{d}+J_{d}-K_{c}$. In this expression we use the rotational symmetry $K_{d}=I_{d}$ of the disk $D$. For control moment gyroscope systems, the nominal operation mode assumes $\left|\omega_{d}\right| \gg \max \left(\left|\omega_{b}\right|,\left|\omega_{c}\right|\right)$, and regulation of the velocity $\omega_{d}$ is performed by the means of a local motor controller. Assuming that this controller provides fast and accurate velocity regulation ensuring $\dot{\omega}_{d} \approx 0$, we will further assume that $\omega_{d}$ is constant and equals to the nominal velocity, see Table I

Moreover, in our hardware the gimbal $C$ is controlled by the means of a servo drive that ensures velocity tracking. In this case, the velocity $\omega_{c}$ can be considered as an input signal. Under these assumptions the above system can be rewritten as

$$
\begin{aligned}
& \dot{q}_{b}= \omega_{b}, \\
& \dot{\omega}_{b}=\frac{\omega_{c}\left(J_{d} \omega_{d} \sin q_{c}+J_{2} \omega_{b} \sin \left(2 q_{c}\right)\right)}{J_{1}+J_{2} \cos ^{2} q_{c}} \\
&+\frac{m l g \sin \left(q_{b}+\frac{\pi}{4}+e\right)}{J_{1}+J_{2} \cos ^{2} q_{c}},
\end{aligned}
$$

III. Linearization-based stabilization

For the system (2), the desired equilibrium is defined as

$$
\Omega_{0}:=\left\{q_{b}=-\frac{\pi}{4}-e, \omega_{b}=0, q_{c}=\frac{\pi}{2}\right\} .
$$


TABLE I: Hardware parameters

\begin{tabular}{|c|c|c|}
\hline Description & Symbol & Value \\
\hline Total mass, kg & $m$ & 2.62 \\
\hline Center of mass to pivot distance, $\mathrm{m}$ & $l$ & 0.13 \\
\hline Moments of inertia, $\mathrm{kg} \cdot \mathrm{m}^{2}$ : & & \\
\hline of the body $B$ & {$\left[I_{b}, J_{b}, K_{b}\right]$} & {$\left[\begin{array}{lll}10 & 13 & 13\end{array}\right] \cdot 10^{-3}$} \\
\hline of the body $C$ & {$\left[I_{c}, J_{c}, K_{c}\right]$} & {$\left[\begin{array}{lll}10 & 2.6 & 9.9\end{array}\right] \cdot 10^{-4}$} \\
\hline of the body $D$ & {$\left[I_{d}, J_{d}, K_{d}\right]$} & {$\left[\begin{array}{llll}5.6 & 11 & 5.6\end{array}\right] \cdot 10^{-4}$} \\
\hline Disk $D$ velocity, $\mathrm{rad} / \mathrm{s}$ & $\omega_{d}$ & 314 \\
\hline Equilibrium position bias, rad & $e$ & unknown \\
\hline
\end{tabular}

In what follows we say that a control law (locally) stabilizes the system (2) if under this control law the point $\Omega_{0}$ is (locally) attractive.

To proceed, it is convenient to define a new state variable as a deviation of the actual position from the desired equilibrium, $x:=\left[\begin{array}{lll}q_{b} & \omega_{b} & q_{c}\end{array}\right]^{\dagger}-\Omega_{0}$. Then the system (2) can be rewritten as

$$
\dot{x}=f(x, u)=\left[\begin{array}{c}
x_{2} \\
f_{2}(x, u) \\
u
\end{array}\right],
$$

where $u=\omega_{c}$ and

$$
f_{2}(x, u)=\frac{u\left(J_{d} \omega_{d} \cos x_{3}-J_{2} x_{2} \sin \left(2 x_{3}\right)\right)+m g l \sin x_{1}}{J_{1}+J_{2} \sin ^{2} x_{3}} .
$$

The state variable $x_{3}$ can be computed through the measurements of the signal $q_{c}$; however, since the offset $e$ is not known, the signal $x_{1}$ can not be computed. Thus, we define the vector of measurements as

$$
y=\left[\begin{array}{c}
q_{b}+\frac{\pi}{4} \\
q_{c}-\frac{\pi}{2}
\end{array}\right]=\left[\begin{array}{c}
x_{1}-e \\
x_{3}
\end{array}\right] .
$$

Then the control goal is to find a control law that stabilizes (3) at the origin using the measurements $y$.

A common practice to stabilize a (sufficiently wellbehaved) nonlinear system is to linearize the system around the desired equilibrium. For the system (3) such equilibrium is given by $x=0, u=0$. Let us define

$$
\begin{aligned}
A & :=\frac{\partial f}{\partial x}(0,0)=\left[\begin{array}{ccc}
0 & 1 & 0 \\
\frac{m g l}{J_{1}} & 0 & 0 \\
0 & 0 & 0
\end{array}\right], \\
B & :=\frac{\partial f}{\partial u}(0,0)=\left[\begin{array}{c}
0 \\
\frac{J_{d} \omega_{d}}{J_{1}} \\
1
\end{array}\right]
\end{aligned}
$$

Then the linearization of (3) around the origin is given by $\dot{x}=A x+B u$.

It is tempting to use the state-feedback static control

$$
u:=-K\left[\begin{array}{lll}
y_{1} & x_{2} & y_{2}
\end{array}\right]=-K x-k_{1} e,
$$

where $K:=\left[\begin{array}{lll}k_{1} & k_{2} & k_{3}\end{array}\right]$ is the gain vector. The problem, however, is that the equilibrium point would be $(A-B K)^{-1} B k_{1} e=\left[\begin{array}{lll}0 & 0 & -\frac{k_{1}}{k_{3}}\end{array}\right]^{\top} e$.

Therefore, the stabilization goal $|x| \rightarrow 0$ is not achieved under the control law (6). In order to overcome the nonzero steady-state body $C$ angle problem, we add an integral action. To this end, we introduce an auxiliary variable $x_{e}$ defined as $\dot{x}_{e}:=0-x_{3}$, where zero stays as the reference for $x_{3}$. Then the extended state-space model is

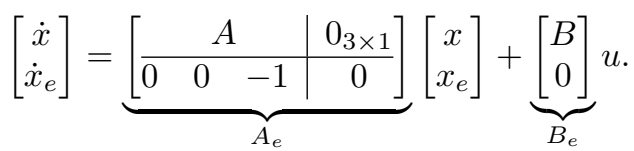

The pair $A_{e}, B_{e}$ is controllable and we can design the extended control law

$$
\begin{aligned}
u & :=-K_{e}\left[\begin{array}{llll}
y_{1} & x_{2} & y_{2} & x_{e}
\end{array}\right]^{\top} \\
& =-K_{e}\left[\begin{array}{ll}
x^{\top} & x_{e}
\end{array}\right]^{\top}-k_{e, 1} e,
\end{aligned}
$$

where $K_{e} \in \mathbb{R}^{1 \times 4}$. Then the closed-loop dynamics is

$$
\left[\begin{array}{c}
\dot{x} \\
\dot{x}_{e}
\end{array}\right]=\left(A_{e}-B_{e} K_{e}\right)\left[\begin{array}{c}
x \\
x_{e}
\end{array}\right]-B_{e} k_{e, 1} e,
$$

and the equilibrium is

$$
\left(A_{e}-B_{e} K_{e}\right)^{-1} B_{e} k_{e, 1} e=\left[\begin{array}{llll}
0 & 0 & 0 & -\frac{k_{e, 1}}{k_{e, 4}}
\end{array}\right]^{\top} e .
$$

Thus, the state $x$ converges to zero, while the integral action $x_{e}$ ensures the equilibrium offset compensation.

However, to implement the control law (7), it is required to estimate the velocity $\omega_{b}$. In the next section we discuss possible velocity observers for the considered system.

\section{Observers design}

A model-based nonlinear observer has been recently proposed in [8] for a class of mechanical systems that are partially linearizable via coordinate changes (PLvCC). The observer can be applied to the mechanical system (1) if it admits a change of coordinates allowing to rewrite the system in such a form that the dynamics is linear in momenta. Then a globally converging exponential momenta observer can be constructed that yields velocity estimation. Unfortunately, despite its simplicity, the considered system does not belong to this class.

A mechanical system with inertia matrix $M$ and $n$ degrees of freedom is partially linearizable via a change of coordinates $(\mathrm{PLvCC})$ if there exists 14 a full-rank matrix $\Psi: \mathbb{R}^{n} \rightarrow \mathbb{R}^{n \times n}$ such that for all $i=1, \ldots, n$ the matrices $\mathcal{B}_{(i)}$ are skew-symmetric, where

$$
\mathcal{B}_{(i)}:=\sum_{j=1}^{n}\left\{\left[\Psi_{i}, \Psi_{j}\right] \Psi_{j}^{\top}\left(M \Psi \Psi^{\top}\right)^{-1}+\frac{1}{2} \Psi_{j i} \Psi \frac{\partial}{\partial q_{j}}\left(\Psi^{\top} M \Psi\right)^{-1} \Psi^{\top}\right\} .
$$

Here $\Psi_{i}$ is the $i$-th column of $\Psi, \Psi_{i j}$ is the element of $\Psi$ with the indices $i$ and $j$, and $\left[\Psi_{i}, \Psi_{j}\right]=\frac{\partial \Psi_{j}}{\partial q} \Psi_{i}-\frac{\partial \Psi_{i}}{\partial q} \Psi_{j}$ is the Lie bracket.

Assuming that regulation of the velocity $\omega_{d}$ is performed by the means of a local motor controller and $\dot{\omega}_{d} \approx 0$, the system (1) can be written in the standard mechanical system form as $M(q) \ddot{q}+C(q, \dot{q}) \dot{q}+G(q)=\tau$, where the inertia matrix is $M(q)=\left[\begin{array}{cc}m_{1}\left(q_{2}\right) & 0 \\ 0 & 1\end{array}\right]$, with $m_{1}\left(q_{2}\right):=\frac{1}{I_{c}+I_{d}}\left(J_{1}+J_{2} \cos ^{2}\left(q_{2}\right)\right)$. Since the PLvCC 
property depends on the inertia matrix only, we are not writing down other terms.

Proposition 1. The inertia matrix $M(q)$ does not belong to the PLvCC class, i.e. there exists no $\Psi$ such that $\mathcal{B}_{(1)}$ and $\mathcal{B}_{(2)}$ are skew-symmetric.

The proof of the proposition is not presented here for the sake of brevity. Since we can not build a model-based nonlinear observer for the system (1), we are considering the reduced system (2). In this section, we present three designs that are suitable for the considered system, namely a linear model-free differentiator, a linear Luenberger's observer, and a nonlinear model-based differentiator.

\section{A. A linear differentiator}

The simplest way to get the velocity estimation is to use a linear differentiation; however, it is well known that differentiation can be noisy at high frequencies. To this end, a common engineering practice for the velocity estimation is to use a low pass filter together with the differentiator. This can be used to reduce the consequences of noise in the signal but care is needed to ensure that the phase lag does not distort the results. For a 2 nd order filter with a time constant $\tau$, the continuous-time transfer function of the model-free differentiator can be written as $G(s)=\frac{s}{(\tau s+1)^{2}}$, yielding the following statespace realization

$$
\begin{aligned}
& \dot{z}_{1}=z_{2}, \\
& \dot{z}_{2}=-\frac{1}{\tau^{2}} z_{1}-\frac{2}{\tau} z_{2}+\frac{1}{\tau^{2}} y_{1}, \\
& \hat{x}_{2}^{l d}=z_{2},
\end{aligned}
$$

where $\hat{x}_{2}^{l d}$ is the estimate of $x_{2}$ provided by the differentiator.

\section{B. A full-order linear state observer}

A full-state model-based linear observer is constructed as a Luenberger's observer for the linearization of the model dynamics at the desired equilibrium:

$$
\dot{\hat{x}}=A \hat{x}+B u+L(y-C \hat{x}), \quad \hat{x}_{2}^{l o}=\hat{x}_{2},
$$

where $\hat{x}_{2}^{l o}$ is the estimate of $x_{2}$ provided by the linear observer, the vector of measurements $y$ is defined in (4), matrices $A$ and $B$ are defined in (5), and

$$
C=\left[\begin{array}{lll}
1 & 0 & 0 \\
0 & 0 & 1
\end{array}\right]
$$

Define the estimation error as $\tilde{x}:=x-\hat{x}$. Then in the absence of the bias, i.e., when $e=0$, the error dynamics becomes $\dot{\tilde{x}}=(A-L C) \tilde{x}$. The gain matrix $L$ is chosen such that the matrix $A-L C$ is Hurwitz. For example, it can be done by pole placement, or as a solution of an optimal estimation problem, e.g., Kalman filter. Then, the asymptotic convergence of $\tilde{x}$ to zero is ensured.

For the case $e \neq 0$ we have $y=C x+\left[\begin{array}{l}1 \\ 0\end{array}\right] e=C x+C_{e} e$ and $\dot{\tilde{x}}=(A-L C) \tilde{x}-L C_{e} e$. Thus, the steady state value
TABLE II: Controller parameters used in experiments.

\begin{tabular}{lcc} 
Description & Symbol & Value \\
\hline LQR gains in & $K_{e}$ & {$[35,4,-1,0.3]$} \\
Time constant in & $\tau$ & 0.02 \\
Observer gains in & $L$ & {$[[20,0],[150,0],[0,20]]$} \\
Parameters in 10$]$ & {$\left[k_{1}, k_{2}, \alpha\right]$} & {$[20,150,0.85]$}
\end{tabular}

of the velocity estimation will differ from zero due to the bias $e$. However, it does not compromise the closed-loop stabilization. Indeed, with the observer, the control action (7) becomes $u=-K_{e}\left[\begin{array}{ll}\hat{x}^{\top} & x_{e}\end{array}\right]^{\top}=-K_{e}\left[\begin{array}{ll}x^{\top} & x_{e}\end{array}\right]^{\top}+$ $K_{e}\left[\begin{array}{ll}\tilde{x}^{\top} & 0\end{array}\right]^{\top}$.

Define $\bar{x}:=\left[\begin{array}{ll}x^{\top} & x_{e}\end{array}\right]^{\top}$. The closed-loop dynamics obeys

$$
\left[\begin{array}{c}
\dot{\bar{x}} \\
\dot{\tilde{x}}
\end{array}\right]=\left[\begin{array}{cc}
A_{e}-B_{e} K_{e} & B_{e} K_{e}\left[\begin{array}{c}
I_{3 \times 3} \\
0_{1 \times 3}
\end{array}\right] \\
0_{3 \times 4} & A-L C
\end{array}\right]\left[\begin{array}{c}
\bar{x} \\
\tilde{x}
\end{array}\right]+\left[\begin{array}{c}
0_{4 \times 1} \\
L C_{e}
\end{array}\right] e .
$$

Then is straightforward to verify that the equilibrium of the closed-loop system satisfies $x=0$, i.e., the integral action compensates the offset being used in the loop with the state observer even if the observer has a steady-state estimation error.

\section{Homogeneous finite-time differentiator}

Following Perruquetti et al. [15], the model-based homogeneous differentiator for the states $x_{1}, x_{2}$ of the system (3) is constructed as

$$
\begin{aligned}
\dot{\hat{x}}_{1} & =\hat{x}_{2}-k_{1}\left\lceil e_{b}\right\rfloor^{\alpha}, \\
\dot{\hat{x}}_{2} & =\frac{u\left(J_{d} \omega_{d} \cos y_{2}-J_{2} \hat{x}_{2} \sin \left(2 y_{2}\right)\right)+m g l \sin y_{1}}{J_{1}+J_{2} \sin ^{2} y_{2}}-k_{2}\left\lceil e_{b}\right\rfloor^{2 \alpha-1}, \\
\hat{x}_{2}^{h d} & =\hat{x}_{2},
\end{aligned}
$$

where $\hat{x}_{2}^{h d}$ is the estimate of $x_{2}$ provided by the homogeneous observer, $e_{b}=\hat{x}_{1}-y_{1}$ and $\left\lceil e_{b}\right\rfloor^{\alpha}=\left|e_{b}\right|^{\alpha} \operatorname{sign}\left(e_{b}\right)$. In 15 it is shown that if $k_{1}$ and $k_{2}$ are such that the polynomial $s^{2}+k_{2} s+k_{1}$ is Hurwitz and $\alpha \in\left(\frac{1}{2}, 1\right]$, then the estimation error $\hat{x}-x$ converges to a vicinity of the origin. More precisely, convergence to the vicinity instead of the finite-time convergence to the origin follows from the replacement of $x_{2}$ and $\sin \left(x_{1}\right)$ in $f_{2}(x, u)$ in $(3)$ with $\hat{x}_{2}$ and $\sin \left(y_{1}\right)=\sin \left(x_{1}+e\right)$, respectively. A more detailed analysis of the size of this vicinity can be performed by the means of Lyapunov function analysis in a similar way as in [16]; however, such a result is rather technical and is not presented here for brevity.

\section{Experiments and comparison}

The hardware for the tests ${ }^{1}$ (shown in Figure 2) is assembled from off-the-shelf components. The hardware parameters are summarized in the Table I] A STM32F746 discovery board was chosen as the main computing unit. We have chosen a small brushless motor to drive the wheel

${ }^{1}$ We have filmed all the experiments, the video is available here: https://youtu.be/0xFanQ0QaEk. 


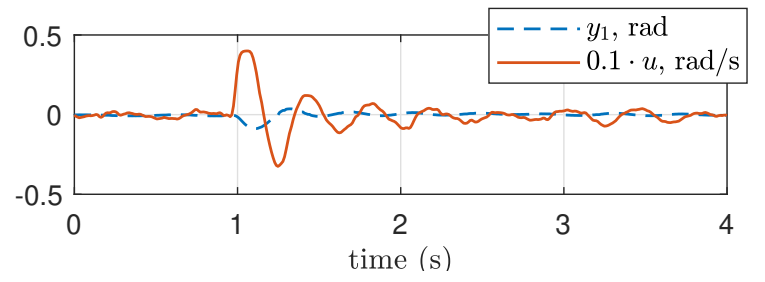

(a) Angle measurement $y_{1}$ and corresponding control $u$

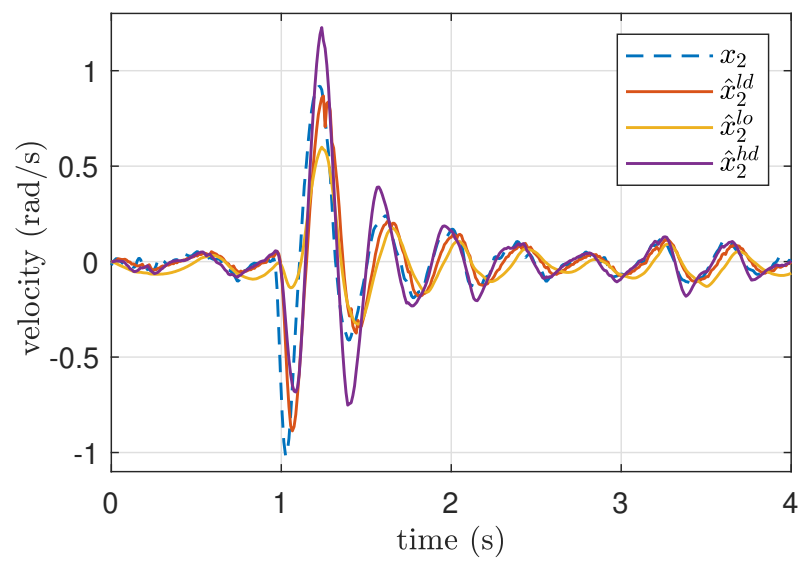

(b) Velocity estimation tuning

Fig. 3: Observers tuning using ground truth measurements of $x_{2}$.

$D$, the frame $C$ is actuated by a Dynamixel MX106$\mathrm{R}$ servo motor. The robot we are currently developing (Figure 1) uses multiple accelerometers to estimate its tilt angles as was proposed in [7], and we rely on soft sensors for velocities estimation. In the case of the 1D pendulum, we use two accelerometers to measure $y_{1}$, and we have installed a fiber optic gyroscope to have the ground truth while comparing the soft sensors.

First of all, since the additional hardware is available, we have implemented the control law (7). Figure 3(a) provides the angle measurements $y_{1}$ and the corresponding control signal $u$. Note that after one second of the experiment an external force was applied for a short period of time. Then we have tuned all three soft sensors to fit the best the measured signal $x_{2}$. Estimated velocities are provided in Figure 3(b). Table II summarizes the control gains as well as the parameters of the soft sensors.

Then we have performed three experiments, replacing the signal $x_{2}$ in the control law (7) by each of the soft sensors we have implemented. The results provided in Figures 4.5 and 6 correspond to the velocity estimators (8), (9) and (10), respectively.

It is easy to see that the non-linear differentiator (10) offers the best behavior. The finite differences introduce an inherent lag into the estimation process, and the system enters into sustained oscillations (Figure 4). A similar behavior can be witnessed (Figure 5 ) for the observer (9). In this case, the oscillations are due to the fact that the linearization does not reflect well the behavior of the

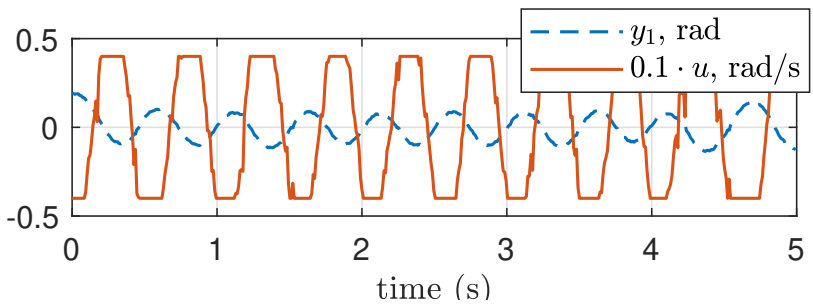

(a) Angle measurement $y_{1}$ and corresponding control $u$

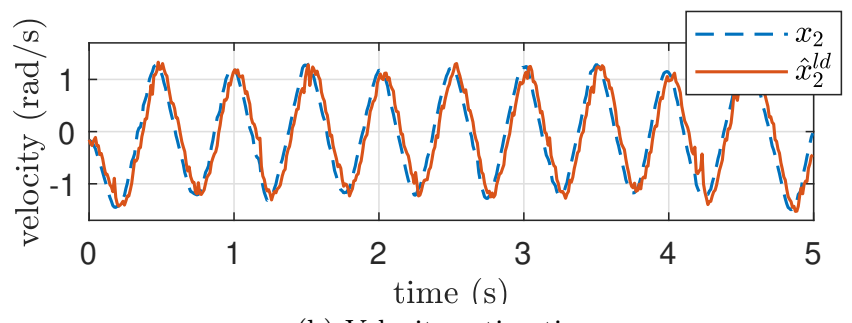

(b) Velocity estimation

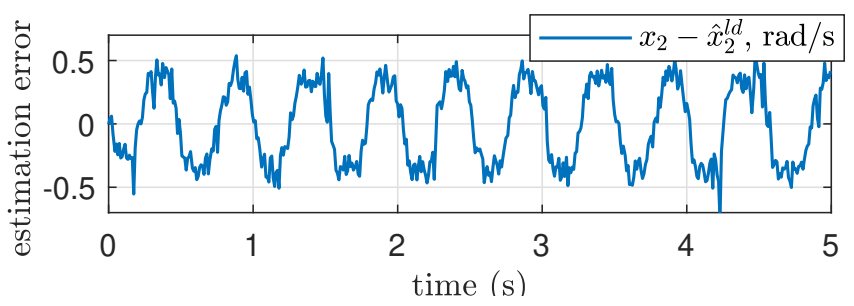

(c) Estimation error

Fig. 4: Closed-loop stabilization using observer (8).

system. In particular, this observer ignores completely the signal $y_{2}$; and thus it is not robust w.r.t external disturbances. An illustration is available in Figure 3(b), where at time step $t \approx 1$ s the linear observer fails completely to estimate the signal $x_{2}$ under a sudden application of an external force.

\section{Conclusion}

We have considered the velocity estimation problem for the control moment gyroscope inverted pendulum case study. Three different velocity observers, namely the linear model-free differentiator, the linear modelbased observer, and the nonlinear model-based differentiator, have been implemented in the hardware and experimentally compared in the pendulum stabilization task. The experimental studies illustrate that the designs that utilize model knowledge outperform the model-free differentiator. Moreover, the nonlinear model-based design provides smaller error than the linear one.

At a further research direction, we intend to apply the designed nonlinear differentiator for the biped system described in Introduction, Fig. 2. At this step, we also intend to incorporate a friction model into the dynamics of the model-based velocity estimator to reduce the modeling errors in the system. 


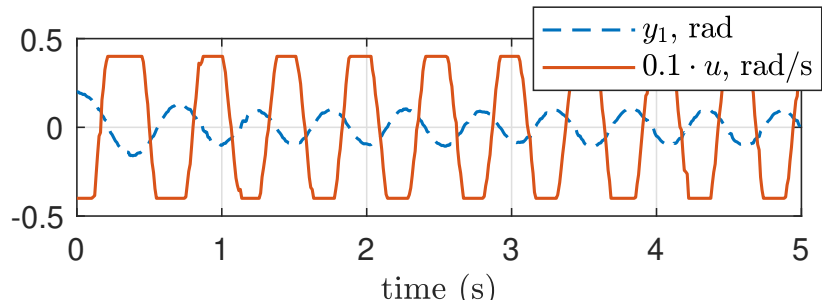

(a) Angle measurement $y_{1}$ and corresponding control $u$

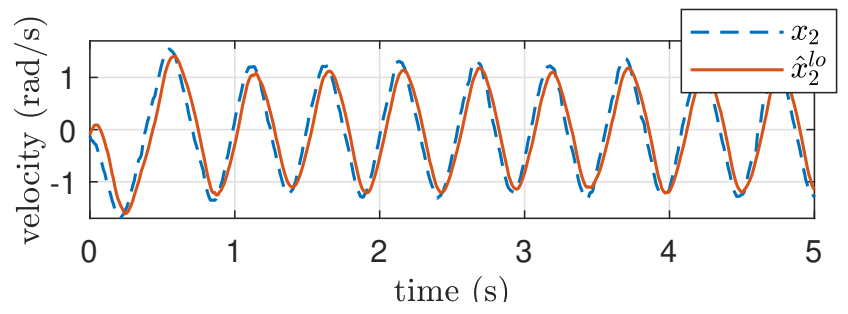

(b) Velocity estimation

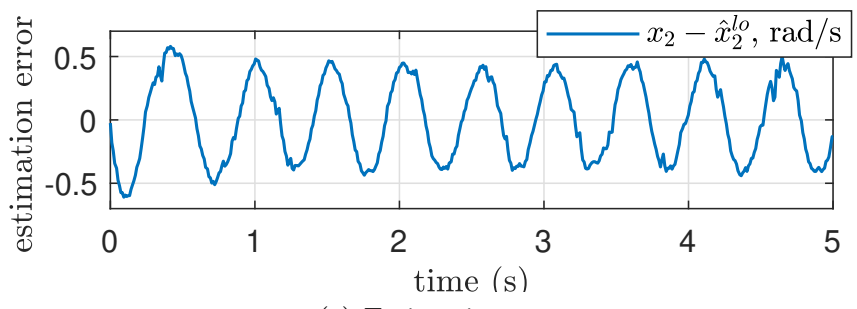

(c) Estimation error

Fig. 5: Closed-loop stabilization using observer (9).

\section{References}

[1] S. Inc., "Anti-roll gyro." [Online]. Available: https://www. seakeeper.com/technology/

[2] S. D. Lee and S. Jung, "Awakening strategies from a sleeping mode to a balancing mode for a sphere robot," International Journal of Control, Automation and Systems, vol. 15, no. 6, pp. 2840-2847, 2017.

[3] J. Chiu and A. Goswami, "Design of a wearable scissoredpair control moment gyroscope (sp-cmg) for human balance assist," in ASME 2014 international design engineering technical conferences and computers and information in engineering conference. American Society of Mechanical Engineers, 2014, pp. V05AT08A023-V05AT08A023.

[4] H. Oya and Y. Fujimoto, "Preliminary experiments for postural control using wearable-cmg," in IECON 2017-43rd Annual Conference of the IEEE Industrial Electronics Society. IEEE, 2017, pp. 7602-7607.

[5] J. A. Paradiso, "Global steering of single gimballed control moment gyroscopes using a directed search," Journal of Guidance, Control, and Dynamics, vol. 15, no. 5, pp. 1236-1244, 1992.

[6] B. Thornton, T. Ura, Y. Nose, and S. Turnock, "Zero-g class underwater robots: Unrestricted attitude control using control moment gyros," IEEE Journal of Oceanic Engineering, vol. 32, no. 3, pp. 565-583, 2007.

[7] S. Trimpe and R. D'Andrea, "Accelerometer-based tilt estimation of a rigid body with only rotational degrees of freedom," in 2010 IEEE International Conference on Robotics and Automation, May 2010, pp. 2630-2636.

[8] S. Aranovskiy, R. Ortega, J. G. Romero, and D. Sokolov, "A globally exponentially stable speed observer for a class of mechanical systems: experimental and simulation comparison with high-gain and sliding mode designs," International Journal of Control, vol. 92, no. 7, pp. 1620-1633, 2019.

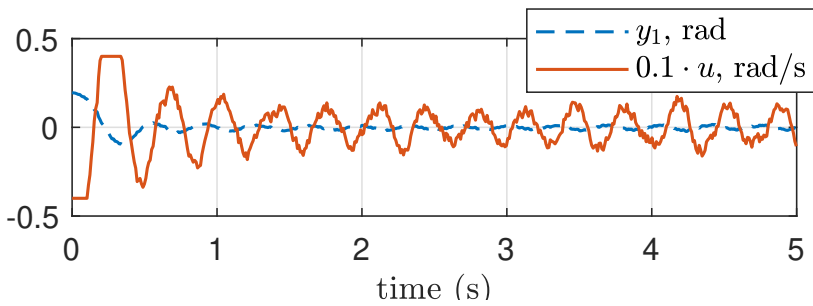

(a) Angle measurement $y_{1}$ and corresponding control $u$

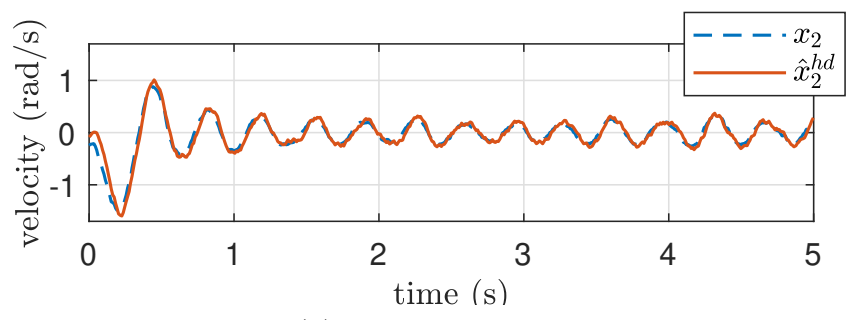

(b) Velocity estimation

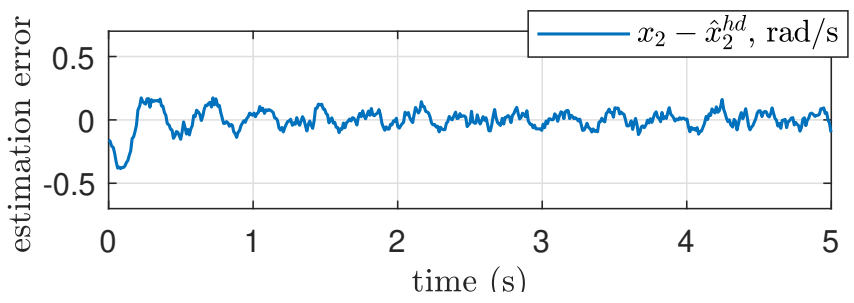

(c) Estimation error

Fig. 6: Closed-loop stabilization using observer 10.

[9] M. W. Spong, P. Corke, and R. Lozano, "Nonlinear control of the reaction wheel pendulum," Automatica, vol. 37, no. 11, pp. $1845-1851,2001$

[10] A. Levant, "Robust exact differentiation via sliding mode technique," Automatica, vol. 34, no. 3, pp. 379-384, 1998.

[11] L. K. Vasiljevic and H. K. Khalil, "Error bounds in differentiation of noisy signals by high-gain observers," Systems \& Control Letters, vol. 57, no. 10, pp. 856-862, 2008.

[12] W. Perruquetti, T. Floquet, and E. Moulay, "Finite-time observers: Application to secure communication," IEEE Transactions on Automatic Control, vol. 53, no. 1, Feb 2008.

[13] ECP (Educational Control Products), "Model 750: Control moment gyroscope," http://www.ecpsystems.com/controls ctrlgyro.htm cited July 2019.

[14] A. Venkatraman, R. Ortega, I. Sarras, and A. van der Schaft, "Speed observation and position feedback stabilization of partially linearizable mechanical systems," IEEE Transactions on Automatic Control, vol. 55, no. 5, pp. 1059-1074, 2010.

[15] W. Perruquetti and T. Floquet, "Homogeneous finite time observer for nonlinear systems with linearizable error dynamics," in 2007 46th IEEE Conference on Decision and Control, Dec 2007, pp. 390-395.

[16] I. Ryadchikov, S. Aranovskiy, E. Nikulchev, J. Wang, and D. Sokolov, "Differentiator-based velocity observer with sensor bias estimation : an inverted pendulum case study," in 11th IFAC Symposium on Nonlinear Control Systems (NOLCOS 19), Vienna, Austria, 2019. 